Cockcroft 07-51

\title{
GDFIDL SIMULATIONS OF NON-LINEAR TAPERS FOR ILC COLLIMATORS *
}

J. D. A. Smith, Lancaster University/Cockcroft Institute, Daresbury, UK $†$ 


\title{
GDFIDL SIMULATIONS OF NON-LINEAR TAPERS FOR ILC COLLIMATORS
}

\author{
J. D. A. Smith, Lancaster University/Cockcroft Institute, Daresbury, UK ${ }^{\dagger}$
}

\begin{abstract}
This paper summarises the GdfidL simulations relating to non-linear collimators, which offer the potential for improved wakefield performance at the ILC. Such collimators provide a further method for understanding the performance of simulation software in this challenging regime. Our results are compared with data from ESA at SLAC.
\end{abstract}

\section{INTRODUCTION}

A collimator must achieve a compromise between being robust enough to withstand asynchronous beam dump, avoiding excessive emittance dilution that reduces the luminosity, and yet be thin enough to avoid creating a shower of particles that would increase the background at the interaction point, adversely affecting the performance of the detectors. The beam impedance is intimately related to the to to wakefields through its Fourier transform. Small apertures with steep transitions can be seen as a large beam impedance mismatch, so adversely effect the wakefields. Consequently, these increase emittance dilution. The challenge of successful collimator design is to achieve small wakefields with a short transition, through optimal selection of collimator geometry and material.

An analogous situation exists in microwave power electronics when a broadband match is to be achieved, but the space is not sufficient to achieve the desired transmission performance using a straight taper [1]. There are a number of solutions which minimize reflections [2] [3], and this paper attempts to apply these and some other novel geometries that have have been inspired by initial experimental results to the collimator design problem.

\section{SIMULATION TECHNIQUE}

A code study [4] has shown that for collimator wakefield EM simulations, GdfidL [5] is a sensible choice as it allows for the large meshes to be prepared and computed on parallel machines, which overcomes restrictions of only having the memory available on a single desktop faced by some software. A recent development in GdfidL allows the user to calculate the field in a localised window around the ultra-relativistic charged bunch distribution only, greatly reducing the size of mesh and memory required, and dramatically speeding up solution time. Comparisons of collimator designs similar to these and rotationally symmetric computations performed in other codes to validate the

\footnotetext{
* This work is supported by the Commission of the European Communities under the 6th Framework Programme "Structuring the European Research Area", contract number RIDS-011899

$\dagger$ j.d.smith@lancs.ac.uk

05 Beam Dynamics and Electromagnetic Fields
}

techniques used are summarised elsewhere in these proceedings [6]. Simulations for each of the collimators were performed with between 2 and 5 different offsets, bunch lengths from $0.3 \mathrm{~mm}$ corresponding to the ILC bunch length to much longer bunch lengths which allow quicker calculation, sometimes a flat section was added, and in all cases a variety of mesh resolutions were used to ensure stability of the results.

\section{GEOMETRY SELECTION}

Preliminary analysis of data taken from experiments on prototype ILC collimators [7] suggests that shallow tapering is less necessary further away from the beam than close to the axis. This is common to most of the novel designs described below.

\section{Exponential Tapers}

In order to match two microwave components of different line impedance at a given frequency, we use a quarter wave resonator, for which one quarter wavelength at the desired frequency is set to the geometric mean of the sections that require matching. This structure is resonant, and is not suitable for the frequency spectrum of a bunch, which is much wider. As this bandwidth improves when the difference in impedance is smaller, the bandwidth of the match can be improved by adding further quarter wave sections, such that each matching quarter wave section has an impedance which is the geometric mean of the sections either side of it. As more sections are added, the increment in the logarithm of the impedance from section to section should follow the binomial coefficients, and as the number of sections becomes large, this approaches a gaussian distribution. Providing the tapering is relatively slow, optimal matching should be achieved by varying the log of the impedance using

$$
\ln \frac{Z(x)}{Z(-\infty)}=\frac{h}{\sqrt{\pi}} \ln \frac{Z(\infty)}{Z(-\infty)} \int_{-\infty}^{x} e^{-h^{2} x^{2}} \mathrm{~d} x
$$

These were numerically calculated, and a look-up table was used to import these data into GdfidL, and engineering drawings. Such a collimator profile was built, and tested with a beam at at SLAC [8]. For this experiment it was known as Collimator 16.

\section{Longitudinally Asymmetric Tapers}

The ILC BDS is a one-pass system, and due to the relatively large bunch to bunch separation, the short-range

D04 Instabilities - Processes, Impedances, Countermeasures 


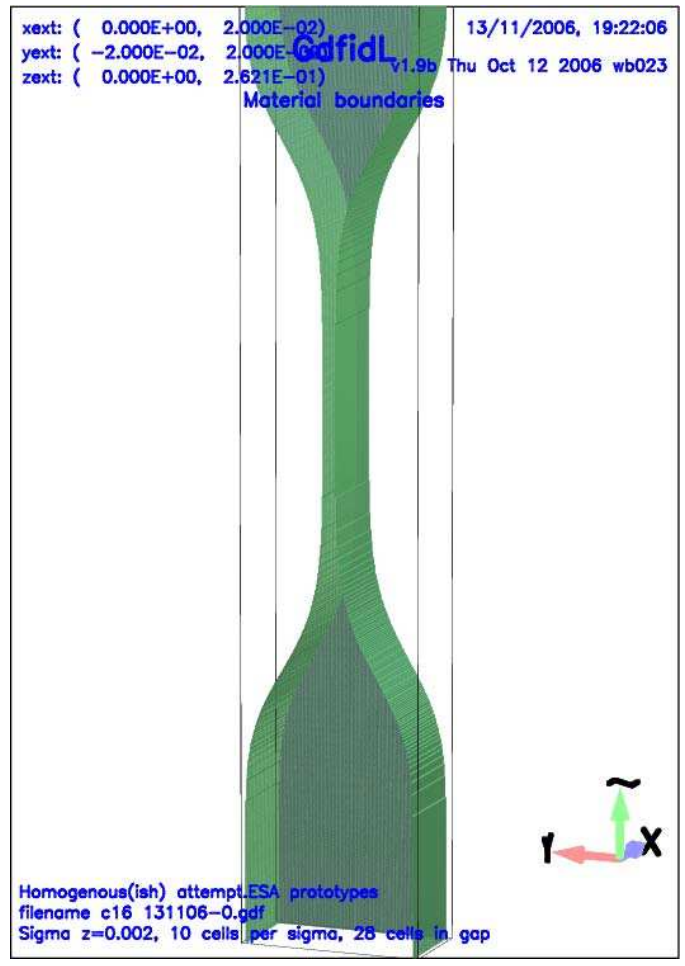

Figure 1: Example of exponentially tapering collimator, beam travels bottom to top.

wakefields concern us far more greatly than the long-range wakefields. It has been proposed that it may be possible to reduce the length of collimator material by exploiting the 'catch up' effect. A test particle will not experience wakefields from particles ahead of them until it has reached a position $z_{c}$, given by

$$
z_{c} \approx \frac{b^{2}-s^{2}}{2 s}
$$

where $s$ is the longitudinal displacement of the test particle from the charge exciting the wake and $b$ is transverse displacement of the obstacle causing the scattering. Taking $s \approx 0.3 \mathrm{~mm}$ for the longitudinal dimension of the bunch, and $z_{c} 1 \mathrm{~km}$ it is clear that any discontinuity within about $0.8 \mathrm{~m}$ of the bunch would be seen, so this is unlikely to be suited to the ILC, however may be worth considering for other accelerators.

\section{Other Novel Structures}

A number of other functions fullfil the requirements of being shallow and smoothly varying in the vicinity of the beam, while being steeper off axis leading to the use of less material. It was unclear whether it was necessary to be smoothly varying at the radius of the beampipe. Some of the other geometries simulated, listed below, were motivated by the desire to determine whether this was necessary:

\section{- Half circle}

05 Beam Dynamics and Electromagnetic Fields

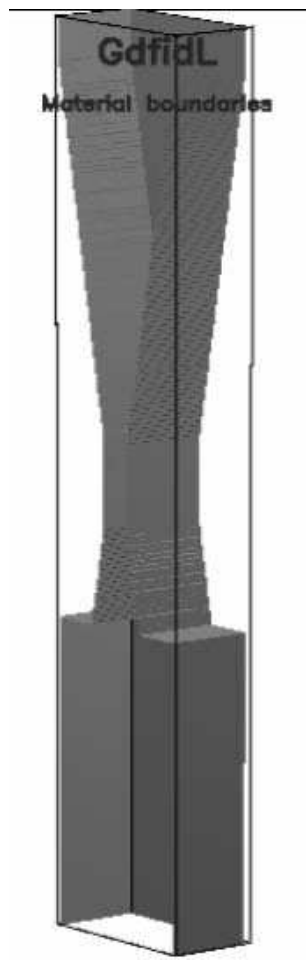

Figure 2: Example of longitudinally asymmetric collimator, beam travels bottom to top.

- Opposing half circles

- Half exponential

- Rectangular from beampipe to $4 \mathrm{~mm}$, then quarter ellipse from 4-1.4mm radius

- Ellipse all the way in from edge boundary

- Half cosine,

- Raised cosine and

- Tanh tapers

- Any of the above, with flat section. Typically 0.021 was chosen as a depth, which corresponds to $0.6 \chi_{0}$ (radiation lengths) in Titanium Alloy.

\section{POST-PROCESSING}

In order to determine the performance a procedure [6] was carried out to factor out mesh stability, and determine an estimate of the kick, and its error. An example calculation for the $0.5 \mathrm{~mm}$ exponential taper is shown in Figure 3.

GdfidL predicts a $2.35 \mathrm{~V} / \mathrm{pC} / \mathrm{mm}$ kick, measured from the linear portion of the plot. The two sets of error bars shown are derived from different estimates of the error of the FDTD algorithm [6].

\section{COMPARISON WITH EXPERIMENTAL MEASUREMENT}

The exponential taper described above was manufactured from OHFC copper, and place in the beamline at the End Station A facility at SLAC, where there is a programme of collimator test studies. While the analysis is not

D04 Instabilities - Processes, Impedances, Countermeasures 


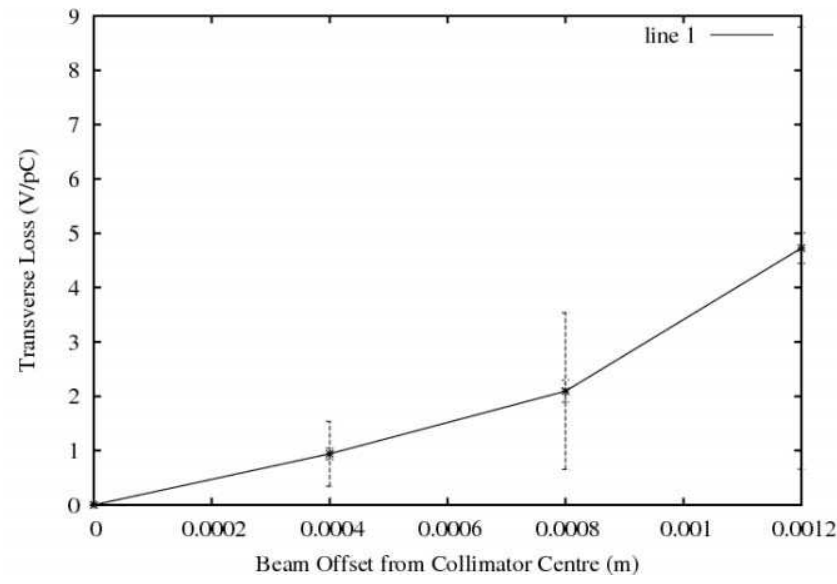

Figure 3: Simulated transverse loss for collimator 16 with bunch length $\sigma_{z}=0.5 \mathrm{~mm}: \kappa_{\perp}=2.35 \pm 1.50 \mathrm{~V} / \mathrm{pC} / \mathrm{mm}$

complete yet, some preliminary results from reference are shown in Figure 4.

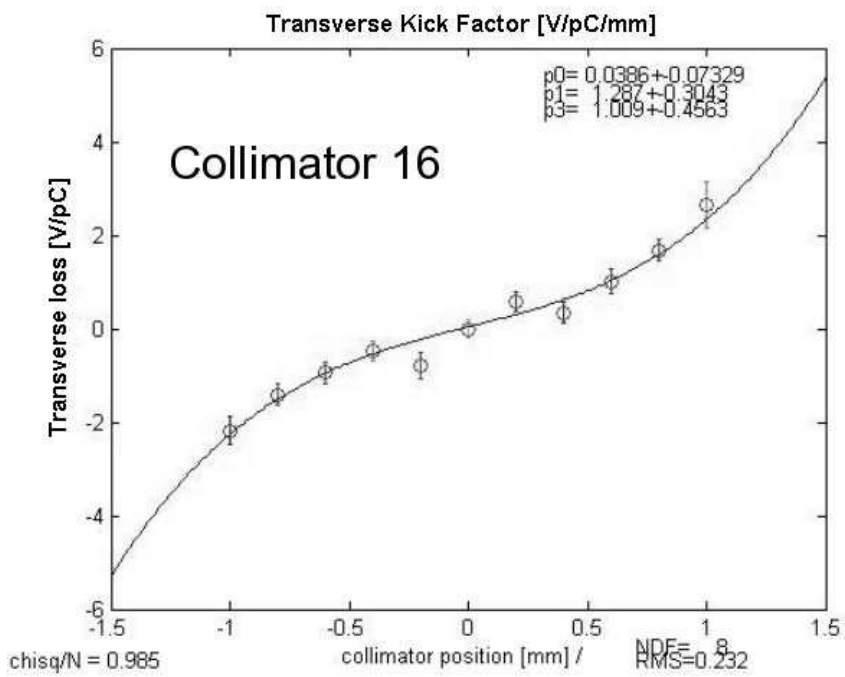

Figure 4: Experimental transverse loss for collimator 16

In this plot the kick is given by the linear term in the cubic fit, in this case $1.3 \pm 0.3 \mathrm{~V} / \mathrm{pC} / \mathrm{mm}$. Regretfully the bunch length is not well defined, although other studies suggest it is around $400 \mu \mathrm{m}$ [9]. Consequently of the values from the simulation would appear to overestimate the error seen greatly, however if one were to assume the error is determined by the difference between simulation value and nearest point, then the data seem consistent, but only on account of the large error in the simulation. Additionally the simulation includes only the geometric component of the kick, whereas the experiment would reflect other effects such as the resistive wall kick and wakefields from surface roughness. Unless these are combined in such a way as to reduce the overall kick, it can only be determined that further work is required to bring GdfidL and experiment into agreement with one another. It is also possible 05 Beam Dynamics and Electromagnetic Fields that the bunch length is longer than assumed above, as the kick for longer bunches in simulations is lower than the $2.35 \mathrm{~V} / \mathrm{pC} / \mathrm{mm}$ quoted above.

\section{CONCLUSION}

A number of collimator geometries have been studied, which show promising wakefield performance, while keeping the length of the proposed collimator structure low. Calculations have been compared with experiment, however there remain unresolved issues in both the understanding of simulation errors and experimental programme before the required level of agreement is achieved. Through utilisation of the cluster at Daresbury, and further time on the escience cluster in Birmingham [10], it should be possible to compute points using a finer mesh and achieve the desired goal of predicting transverse wakefield kicks to within $10 \%$. Nevertheless, data is compatible between simulation and experiment, and with further study it is anticipated the uncertainties will decrease.

\section{REFERENCES}

[1] T. Moreno, "Microwave Transmission Design Data", McGraw-Hill, New York (1948) pp.51-55

[2] R. W. Klopfenstein, "A Transmission Line Taper of Improved Design”, Proc. IRE Vol. 44, (1956) pp.31

[3] L. Solymar, "Spurious Mode Generation in Nonuniform Waveguide", IRE Transactions on Microwave Theory and Techniques Vol. 7 (1959) pp.379

[4] C.D. Beard and J. D. A. Smith, "Numerical Calculations of Collimator Insertions”, EPAC'06, Edinburgh, June 2006, p. 709

[5] http://www.gdfidl.de

[6] J. D. A. Smith and C. J. Glasman, "Computations of Wakefields in the ILC Collimators", these proceedings.

[7] N.K.Watson et al., "Direct Measurement of Geometric and Resistive Wakefields in Tapered Collimators for the International Linear Collider", EPAC'06, Edinburgh, June 2006, p. 697

[8] S. Molloy et al., "Measurements of the Wakefields Due to the Varying Collimator Characteristics", these proceedings.

[9] S. Molloy et al, "Picosecond Bunch length and Energy-z Correlation Measurements at SLAC's A-Line and End Station A.", these proceedings.

[10] http://www.ep.ph.bham.ac.uk/cluster/ 